\title{
Evidências de validade da escala de locus de controle da preservação e conservação ambiental
}

\section{Evidence of validity of the locus scale for environmental preservation and conservation control}

\author{
Marcela Nunes Aguiar (orcid.org/0000-0001-5659-4707)' \\ Marcos Aguiar de Souza (orcid.org/0000-0001-8831-9056) ${ }^{2}$ \\ Hermes de Andrade Jr. (orcid.org/0000-0002-3667-1354) \\ Pedro P. Pires (orcid.org/0000-0001-8831-9056) ${ }^{4}$
}

\begin{abstract}
Resumo
A questão ambiental é um campo de grande importância na atualidade, principalmente no que tange aos estudos sobre valores pró-ambientais. Uma interação normalmente investigada consiste na relação entre conexão com a natureza e locus de controle, especialmente considerando a crença por parte do indivíduo de que suas ações sejam importantes para a conservação do meio ambiente. Participaram do estudo 898 estudantes universitários, sendo 452 (49,8\%) homens, 456 (50,2\%) mulheres de idade média, de 28,87 (DP=8,90) anos, do estado do Rio de Janeiro. Para fins de validade interna, foram empregados procedimentos de análise fatorial. A escala foi analisada também junto à medida de conexão com a natureza, para oferecer uma evidência inicial de validade concorrente. Conexão com a natureza foi mensurada por meio da escala proposta por Mayer e Frantz (2004). A Escala de Locus de Controle da Preservação Ambiental foi desenvolvida neste estudo. Considerando os resultados obtidos, é possível afirmar uma qualidade satisfatória da medida desenvolvida.
\end{abstract}

Palavras-chave: Locus de controle. Preservação ambiental. Conservação ambiental. Atitudes. Valores ambientais.

\begin{abstract}
The environmental issue is a field of great importance today, especially with regard to studies on pro-environmental values. A commonly investigated interaction is the relationship between connection with nature and locus of control, especially considering the individuals' beliefs that their actions are important for the conservation of the environment. The study included 898 university students, which $452(49.8 \%)$ were men, $456(50.2 \%)$ were women of average age, of $28.87(\mathrm{SD}=8.90)$ years old, from the state of Rio de Janeiro. For purposes of internal validity, factor analysis procedures were used. The scale was also analyzed together with the measure of connection with nature, to

\footnotetext{
${ }^{1}$ Universidade Federal Fluminense, Niterói, Brasil, E-mail: marcelaa@id.uff.br.

${ }^{2}$ Universidade Federal do Rio de Janeiro, Rio de Janeiro, Brasil, E-mail: maguiarsouza@uol.com.br.

${ }^{3}$ Universidade de Vigo (Doc CREA S2i), Pontevedra, Espanha. E-mail: handradejunior@gmail.com.

${ }^{4}$ Universidade Federal do Rio de Janeiro, Rio de Janeiro. Brasil, E-mail: ppires85@gmail.com.
} 
offer initial evidence of concurrent validity. Connection with nature was measured using the scale proposed by Mayer and Frantz (2004). The Locus Scale for Environmental Preservation Control was developed in this study. Considering the results obtained, it is possible to affirm a satisfactory quality of the measure developed.

Keywords: Locus of control. Environmental preservation. Environmental conservation. Attitudes. Environmental values.

Os esforços têm sido notados, mas revelam-se ainda não suficientes para reverter o caos que a questão ambiental anuncia para um futuro próximo. Nos termos de Quinn e Burbach (2008), o século XXI será crítico para o ser humano e para todos os sistemas naturais. Mudança global do clima, poluição do ar e da água, práticas equivocadas de uso da terra e perda da biodiversidade são outras questões presentes. Constata-se que os seres humanos, por meio de suas atividades industriais e agropecuárias, estão alterando a estrutura física, biótica e socioeconômica do planeta e intensificando os processos naturais que se afastam do equilíbrio e que alteram a capacidade de resiliência dos ecossistemas ou da biota como um todo.

Nesse quadro, a preocupação com o meio ambiente vem seguida por uma série de estudos que podem ser acessados utilizando-se expressões, como: educação ambiental (e.g. Damerell, Howe, \& Milner-Gulland, 2013), comportamento pró-ambiental (e.g. Steg, Bolderdijk, Keizer, \& Perlaviciute, 2014), consciência ambiental (e.g. Flammer, 2013), conexão com a natureza (e.g. Capaldi, Dopko, \& Zelenski, 2014), desenvolvimento sustentável (Le Blanc, 2015), responsabilidade socioambiental corporativa (e.g. Tan-Mullins \& Mohan, 2013), entre outros, sendo que a lista de descritores é extensa, bem como a de autores. Essa diversidade talvez esteja relacionada ao aspecto interdisciplinar e transversal do tema, uma vez que questões ambientais estão, literalmente, em todas as áreas de atividade humana.

De acordo com o relatório Global Environment Outlook $6^{\text {th }}$ (GEO-6, ONU, 2018), áreas urbanas continuam em crescimento na América Latina com a expectativa de atingir uma população total de 567 milhões de pessoas até 2025, sendo acompanhada por uma redução persistente da qualidade do ar. Questões como a pobreza e problemas de infraestrutura também têm representado um elevado risco ambiental. De acordo com a 
Global Footprint Network (2018), a partir de agosto de 2018, a humanidade começa a consumir mais recursos do que o ritmo de renovação ambiental permite. Muitas vezes, a população em geral deixa de perceber os agravos em situação de pré-crise. Somente nos momentos de crise, a população e também as estruturas públicas que lidam com a defesa civil, de catástrofes e proteção civil de vários países como o Brasil acabam por alarmar-se. Isso se deve à falta de preparação para lidar com fenômenos de alta magnitude ambiental como rotina de contingência. Também há que levar em consideração os custos de uma consequência ambiental de monta, além dos recursos materiais, não fungíveis e vitais, desperdiçados.

Outro aspecto relevante para abordar a problemática ambiental é a crença. Pessoas e organizações são fortemente motivadas por suas tradições, herança e cultura. A crença é importante!!! Em face das dificuldades cotidianas que se acentuam com a crise de várias áreas, em meio à impunidade e ocasiões que culminam em situações de caos social, buscamos por variáveis capazes de influenciar e predizer o comportamento pró-ambiental.

O objetivo do presente estudo é investigar se existe a crença por parte do indivíduo de que suas ações sejam importantes para a preservação ou para a conservação do meio ambiente em um gradiente de conexão com a natureza (comportamento pró-ambiental). $\mathrm{O}$ artigo é apresentado em três seções, a saber: (1) o referencial teórico, que apresenta os conceitos de locus de controle e do locus de controle de conexão com a natureza; (2) o método e os procedimentos; (3) os resultados, sua discussão e as considerações finais.

\section{Locus de controle}

A relevância do tema pode ser atestada pelo número de estudos que têm sido publicados nos últimos anos. O locus de controle é um construto que foi introduzido na psicologia por Rotter (2011), utilizado para categorizar a motivação básica das pessoas em relação às orientações e percepções sobre o quanto de controle possuem sobre as diversas condições de suas vidas. Assim, a pessoa pode se perceber como controladora dos acontecimentos ou como sendo esses acontecimentos controlados por fatores externos a ela (Coleta, 1987; VandenBos, 2015). Alguns sujeitos creem que são donos de seu próprio destino, outros se percebem como joguetes do acaso, achando que tudo que Ihes acontece é 
resultado da sorte ou da causalidade. O primeiro tipo tende a ter o "locus de controle interno" e o segundo o "locus de controle externo" (Robbins, 2005).

Para melhor entendimento do fenômeno, várias escalas foram elaboradas. Inicialmente, uma das mais utilizadas era a Escala de Locus de Controle Interno-Externo de Rotter, também conhecida como Escala I-E (internalidade-externalidade) de Rotter (Rotter, 2011), unifatorial, composta por 23 itens válidos e seis de distração (Coleta, 1987).

Alguns pesquisadores questionaram a unidimensionalidade da Escala de Rotter, indicando que a externalidade poderia ser decomposta em outros campos de controle, para além do mero acaso. Hanna Levenson (1981) desenvolveu uma nova escala para medida do fenômeno, com a premissa de que a externalidade não necessariamente devia ser ruim ou indesejável. Assim, dividiu a externalidade em duas, pois entendia que havia diferença entre pessoas que acreditam no poder de outras sobre si e pessoas que percebem o mundo como imprevisível e incontrolável. Dessa forma, construiu uma escala para medir três dimensões do controle: I (internalidade), P (externalidade outros poderosos) e C (externalidade-acaso) (Coleta, 1987).

A subescala I mede o quanto as pessoas acreditam que têm controle sobre suas próprias vidas (dimensão pessoal). A subescala P mede a percepção de que o controle estaria nas mãos de outras pessoas poderosas (dimensão social). Já a subescala C está relacionada com a percepção de ser controlado pela sorte, acaso ou destino (dimensão impessoal). Levenson (1981) destaca que o diferencial de sua escala está no fato de que a externalidade não precisa ser associada a algo negativo ou ruim, pois pode estar relacionada à realidade da pessoa, como no caso de presidiários ou, até mesmo, dos militares, que vivem sob um sistema disciplinar rígido e alicerçado no respeito à hierarquia (Coleta, 1987; Tamayo, 2012).

A Escala Multidimensional de Locus de Controle de Levenson (1981) é um instrumento composto originalmente por 24 itens, sendo oito para cada dimensão (I, P e C), respondidos em uma escala tipo Likert de seis pontos, desde "discordo fortemente" (-3) até "concordo fortemente" (+3). A tradução e a adaptação ao contexto brasileiro foram feitas por Coleta (1987), que validou o instrumento para o contexto nacional (Tamayo, 2012). 
O locus de controle é considerado como um dos conceitos mais influentes para a psicologia contemporânea, pois explica e descreve as crenças mais ou menos estáveis, pelas quais a pessoa estabelece a origem, o centro do controle de seus eventos e do seu próprio comportamento, segundo Clarke, Probst, Guldenmund e Passmore (2015). O fenômeno vai além do escopo cognitivo-perceptivo-emocional e representa uma forma de existência, pela qual a pessoa não somente explica, mas também define e estrutura seu próprio destino (Tamayo, 2012).

Pesquisadores do comportamento organizacional, como Robbins (2005), mostraram em seus estudos que pessoas do grupo dos "externos" (alta externalidade), normalmente, são mais insatisfeitas com o trabalho, têm maior absenteísmo e são mais alienadas quanto às normas. Em comparação com as pessoas do grupo dos "externos", os internos (alta internalidade) costumam tomar mais atitudes e tendem a ser mais bem sucedidos e satisfeitos no trabalho. Além disso, buscam mais ativamente informações antes da tomada de decisão, são mais motivados para as conquistas e se esforçam mais para ganhar controle sobre seu ambiente. Assim, esse grupo se dá melhor em tarefas mais sofisticadas e funções executivas, que exigem iniciativa e processamento de complexas informações e funções executivas (Robbins, 2005).

Zedeck (2011) apresenta uma série de resultados de estudos sobre o tema. Indivíduos internos lidam melhor com o estresse e têm melhor saúde. A internalidade também está associada com a crença de que resultados podem ser controlados, assim, indivíduos são mais ativos ao buscar maneiras de lidar com situações negativas quando elas ocorrem. O fenômeno da internalidade correlaciona-se positivamente com bem-estar (incluindo bem-estar mental e saúde física) e negativamente com burnout, por meio de processos cognitivos, autoconceito, motivação e estratégias de enfretamento (coping).

Segundo Bandeira et al. (2005), pesquisas apontam que a internalidade estaria positivamente correlacionada com comportamento assertivo. Indivíduos mais assertivos são mais orientados internamente e apresentam menor frequência de sintomas de doenças relacionadas ao estresse, em relação aos demais.

No Brasil, a maioria dos estudos sobre o locus está relacionada à adaptação da escala de Levenson (1981) ao contexto de jovens do ensino superior. O instrumento elaborado por 
Levenson (1981) foi selecionado como o mais adequado para o presente estudo, pela multidimensionalidade, pela ampla utilização (internacional e nacional), pelo perfil de faixa etária de nossos participantes - com média de 20,5 anos - (assemelhada à dos estudantes universitários brasileiros) e porque será aplicada ao ensino superior (assim como outros estudos nacionais).

\section{Locus de controle e conservação ambiental}

Para Corral-Verdugo (2012), a Psicologia Positiva e a psicologia da sustentabilidade estão interessadas no estudo e na promoção de relações humanas positivas, altruísmo, instituições sustentáveis, responsabilidade, felicidade, comportamento autorregulado, autonomia e bem-estar psicológico, entre outras emoções positivas. Essa relação é ainda mais clara diante da constatação de que as emoções positivas estão entre os antecedentes deposicionais que promovem o comportamento ambiental ou sustentável.

Quinn e Burbach (2008) destacam a necessidade de se considerar simultaneamente o papel do comportamento pró-ambiental, seja individual ou de populações inteiras, e as melhores práticas de gestão, seja na agricultura ou em outros setores. De qualquer forma, qualquer modelo relativo ao ambiente deve considerar fatores pessoais, culturais, educacionais, bióticos, físicos, políticos, econômicos e institucionais. É importante perceber a necessidade de uma abordagem horizontal e interdisciplinar na elaboração de modelos relativos ao meio ambiente, visto que suas variáveis, atividades, impactos, danos e conflitos são mapeados pelo olhar antrópico, o que, consequentemente, leva à necessidade de englobar os aspectos que envolvem as relações humanas. Tendo isso em vista, faz-se mister afirmar que nenhum modelo único será eficaz para o ambiente.

Kortenkamp e Moore (2001) destacam dois aspectos relativos ao comportamento pró-ambiental: o antropocentrismo e o ecocentrismo. O antropocentrismo considera que os seres humanos são a mais importante forma de vida e que outros seres vivos são importantes apenas na medida em que podem ser úteis para os seres humanos. A natureza tem sua importância apenas porque sua degradação ou preservação pode prejudicar ou beneficiar os seres humanos. Em uma visão ecocêntrica, por sua vez, o equilíbrio e 
capacidade de resiliência do ambiente (entorno) não devem ser alterados, o que inclui o ser humano, fatores bióticos (fauna e flora) e abióticos (como os cursos d'água, rochas, minerais, relevo). Nessa visão, o ambiente (entorno), em seu aspecto mais amplo, deve ser valorizado por possuir um valor intrínseco, independentemente de sua utilidade para os seres humanos. Todos os componentes do meio, assim como as espécies vegetais e espécies animais, são vistos com igual valor aos seres humanos.

Karch (2002) acrescenta ainda a visão biocêntrica, na qual se defende que todos os organismos vivos têm direito à vida. Assim, as espécies vegetais e animais são vistas como tendo o mesmo valor e direito à vida que o ser humano. A visão ecocêntrica se aproxima da conexão com a natureza, indicando um posicionamento de valorização da natureza e de sintonia com o mundo natural. Considera-se ainda que é o posicionamento que melhor atende aos anseios daqueles que estão voltados para a defesa do ambiente.

Em outro detalhe de abordagem, a visão antropocêntrica aproxima-se da Conservação Ambiental, que significa proteger os recursos naturais com utilização racional, garantindo a sustentabilidade dos mesmos. A visão ecocêntrica aproxima-se da Preservação Ambiental, pois obriga a proteção integral, ou seja, o recurso permanece intacto e sem interferência da ação humana.

De acordo com Capaldi, Dopko e Zelenski (2014), diversos estudos têm evidenciado diferenças consistentes em termos de personalidade, de atitudes, de comportamento e bem-estar em função da conexão ou não com a natureza, evidenciando o caráter positivo de se estar ligado ao mundo natural. Adicionalmente, tais autores defendem que a conexão com a natureza prediz de forma consistente o comportamento pró-ambiental (grifo nosso).

Trabalhamos dentro dessa perspectiva, pois percebemos a conexão com a natureza associada a diversos indicativos de saúde, bem-estar e qualidade de vida. Tal evidência pode ser obtida na literatura, apesar dos estudos ainda escassos relacionando temas da psicologia positiva com a conexão com a natureza e o comportamento pró-ambiental, tanto como variável independente quanto variável dependente (Corral-Verdugo, 2012; Howell, Dopko, Passmore, \& Buro, 2011 ; Nisbet, Zelenski, \& Murphy, 2011).

Dessa forma, a organização do estudo baseado no locus de controle o direciona para a percepção individual do que seja conservação e preservação ambientais e para como a 
crença de tais megafatores ecológicos sinalizam o comportamento pró-ambiental. O artigo é apresentado em cinco seções, a saber: (1) o referencial teórico, que apresenta os conceitos de locus de controle e do locus de controle de conexão com a natureza; (2) o modelo explicativo; (3) o método e os procedimentos; (4) os resultados e sua discussão com a literatura; (5) as considerações finais.

\section{Método}

\section{Participantes}

Participaram do estudo 898 estudantes universitários de diferentes cursos de uma universidade privada brasileira, sendo $452(49,8 \%)$ homens e $456(50,2 \%)$ mulheres. A idade dos participantes variou de 18 a 70 anos (média de 28,87 anos e desvio padrão de 8,90 anos), pertencentes a diversos cursos universitários do estado do Rio de Janeiro.

\section{Instrumentos}

A conexão com a natureza foi mensurada com a utilização do instrumento do mesmo nome, de autoria de Mayer e Frantz (2004), validada para amostras brasileiras por Pessoa et al. (2016). Trata-se de um instrumento do tipo Likert, composto por 14 itens distribuídos em um único fator, com respostas variando de 1 (discordo totalmente) a 5 (concordo totalmente).

A Escala de Locus de controle da preservação ambiental (ELCPA) foi desenvolvida para fins do presente estudo. $O$ instrumento contou, inicialmente, com 20 itens em formato tipo Likert de cinco pontos, variando de 1 (discordo totalmente) a 5 (concordo totalmente), sendo dez indicativos de crenças de que o comportamento individual pode causar prejuízo ao meio ambiente (internalidade) e dez relativos à compreensão de que o dano ao meio ambiente é ocasionado por ações coletivas, envolvendo grandes organizações, municípios, estados ou mesmo o país como um todo. Assim, existe a compreensão de que as ações do cidadão comum não produzem efeitos significativos sobre o ambiente (externalidade). Os itens foram construídos a partir do debate entre os autores do estudo, da consulta à literatura 
especializada e da análise de seis juízes, especialistas da área. Assim, a Escala de Locus de Controle da preservação ambiental foi construída com o intuito de obtenção de um instrumento bifatorial, mensurando a internalidade e a externalidade do fenômeno.

\section{Procedimentos}

Os instrumentos foram aplicados aos participantes em formato digital. Após a leitura do termo de consentimento livre e esclarecido, caso concordassem em participar da pesquisa, marcariam "sim", tendo acesso ao questionário. Se fosse assinalada a alternativa "não", a participação na pesquisa era finalizada. Todos os preceitos éticos foram seguidos para a realização do estudo.

Todos os preceitos éticos de pesquisa foram seguidos. Os respondentes poderiam interromper a participação na pesquisa tão logo desejassem, sendo garantido total sigilo em relação às respostas apresentadas.

\section{Resultados e Discussão}

Antes da realização da análise de dados, a amostra foi dividida em dois grupos, para fins de realização da Análise Fatorial Exploratória (AFE) e Análise Fatorial Confirmatória (AFC). Cada grupo contou, então, com 449 participantes. O total dos participantes foi considerado para fins da testagem do modelo de influência do locus de controle da preservação ambiental sobre a conexão com a natureza, utilizando a modelagem de equações estruturais (MEE).

Em seguida, foi realizada a AFE dos principais eixos com rotação oblimin. O índice KMO foi de 0,87 e o teste de esfericidade de Bartlett foi significativo ao nível de 0,001. Tais resultados permitem afirmar pela adequação de utilização da AFE nos itens da escala (Hair, Black, Babin, Anderson, \& Tatham, 2006). Foram retidos 12 itens que apresentaram carga fatorial igual ou superior a 0,40, igualmente distribuídos em dois fatores, que juntos explicaram $44,78 \%$ da variância observada. 


\section{Tabela 1}

Itens, carga fatorial e consistência interna da escala de locus de controle da preservação ambiental

Itens
Internalidade
Externalidad
(alfa $=$
e $($ alfa $=$
0,759)
0,781)

17. Os seres humanos podem evitar danos ao ambiente

0,72

se forem cuidadosos e conscientes das consequências de suas ações.

14. Pequenas ações, como não recolher adequadamente

o óleo usado e o descarte inadequado de lixo, causam prejuízo ao meio ambiente.

11. Em sua maioria, os danos ao ambiente ocorrem em função de ações que poderiam ser evitadas.

10. A legislação deveria ser mais exigente com as

pessoas, quando estas cometem atos prejudiciais ao ambiente.

9. Em sua maioria, os danos ambientais ocorrem devido aos descuidos do ser humano.

8. De maneira geral, as pessoas costumam adotar algum tipo de comportamento que é prejudicial ao ambiente.

15. A maioria dos danos ambientais pode ser atribuída a uma mudança natural do planeta.

16. Em sua maioria, os danos ao ambiente são causados por fatores que estão fora do controle do ser humano. 
13. As alterações ambientais (catástrofes climáticas, seca, aquecimento global etc) ocorrem por uma questão 0,56 de destino, acaso ou sorte.

2. O que tem sido chamado de dano ao meio ambiente, na verdade, é um processo natural de mudança da 0,53 natureza.

5. Os danos ambientais e as mudanças climáticas ocorrem por fatores que vão além das ações humanas.

3. O cidadão comum não tem tanta capacidade de prejudicar o meio ambiente como tem sido veiculado 0,42 pela mídia.

Em seguida, foram realizados os procedimentos de AFC, utilizando o segundo grupo constituído a partir da divisão dos participantes do estudo. Foram considerados no modelo os indicadores $\mathrm{X}^{2}, \mathrm{X}^{2} / \mathrm{gl}$, RMR, GFI, AGFI, CFI e RMSEA para avaliação do ajuste do modelo (Byrne, 2001; Hair et al., 2006; Kaplan, 2000; Ullman, 2007).

A razão do $X^{2}$ (qui-quadrado) pelo grau de liberdade avalia a pobreza do ajustamento. Assim, quanto menor o valor, melhor. Em geral, o modelo ideal possui o valor de 1 , sendo aceitável um valor de até 5 . Valores superiores a 5 indicam um modelo muito empobrecido, que não deve ser aceito.

O Root Mean Square Residual (RMR) indica o ajustamento do modelo teórico aos dados, quanto mais próxima de zero a diferença entre os dois modelos, mais próximo estaremos de um modelo perfeito. Um modelo bem ajustado possui valor de RMR menor que 0,05 .

O Goodness-of-Fit Index (GFI) e o Adjusted Goodness-of-Fit Index (AGFI) são índices similares ao $\mathrm{R}^{2}$ em regressão múltipla. Assim, indicam a proporção de variância-covariância nos dados explicada pelo modelo. Estes variam de 0 a 1 , sendo considerados satisfatórios valores acima de 0,80 . 
O Comparative Fit Index (CFI) compara, de forma geral, o modelo estimado e o modelo nulo, considerando valores mais próximos de 1 como ideais. Entretanto, valores até 0,9 são considerados satisfatórios. A Root-Mean-Square Error of Approximation (RMSEA), com seu intervalo de confiança de 90\% (IC90\%), é considerada um indicador de "maldade" de ajuste, isto é, valores altos indicam um modelo não ajustado. Assume-se como ideal que o RMSEA se situe entre 0,05 e 0,08, aceitando-se valores de até 0,10 .

\section{Figura 1}

Análise Fatorial Confirmatória da Escala de Locus de Controle da Preservação ambiental

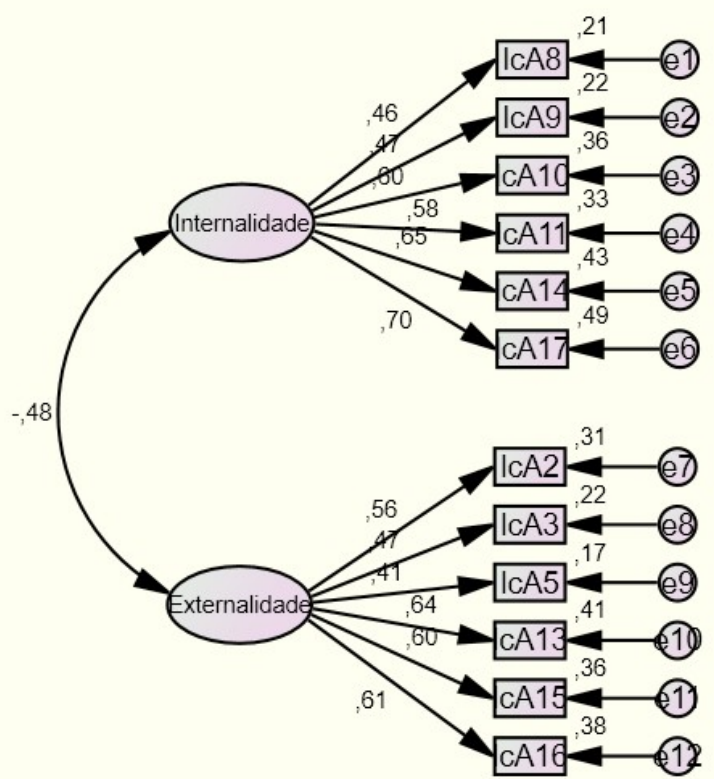

Tabela 2

Índices de ajuste do modelo da Escala de Locus de Controle da Preservação ambiental

\begin{tabular}{ccc}
\hline Indicadores & Valor obtido & Valor ideal \\
\hline $\mathrm{X}^{2}$ & 94,725 & - \\
$\mathrm{X}^{2} / \mathrm{gl}$ & 1,787 & Até 5 \\
$\mathrm{RMR}$ & 0,030 & Menor que 0,05 \\
$\mathrm{GFI}$ & 0,967 & Acima de 0,80 \\
$\mathrm{AGFI}$ & 0,951 & Acima de 0,80 \\
CFI & 0,959 & Próximo a 1
\end{tabular}


RMSEA $\quad 0,042(0,028-0,055) \quad$ Menor que 0,10

Considerando conjuntamente os resultados obtidos com a AFE, AFC e análise da consistência interna da escala (alfa de Cronbach), é possível afirmar validade estrutural da mesma. É interessante observar que não foram necessários ajustes em termos do índice de modificação. O próximo passo é a busca por indicadores de validade que vão além da evidência de consistência interna da escala. É nesse sentido que foi utilizada a técnica de MEE para fins de análise de um modelo explicativo da influência do locus de controle da preservação ambiental sobre a conexão com a natureza, permitindo, assim, obter indicativo de validade concorrente para a ELCPA.

A lógica assumida é que os indivíduos que acreditam que seus atos possam ter repercussões sobre a natureza e, portanto, sobre a preservação ambiental, apresentaram índices mais elevados de conexão com a natureza. Dito de outra forma, o locus interno de preservação ambiental tem uma influência positiva sobre a conexão com a natureza, sendo tal relação de ordem inversa, quando considerado o locus externo de preservação ambiental. Assim, foi testado o modelo de influência do locus de controle da preservação ambiental sobre a conexão com a natureza. 
Figura 2

Modelo métrico completo considerando as variáveis latentes de conexão com a natureza e locus de controle

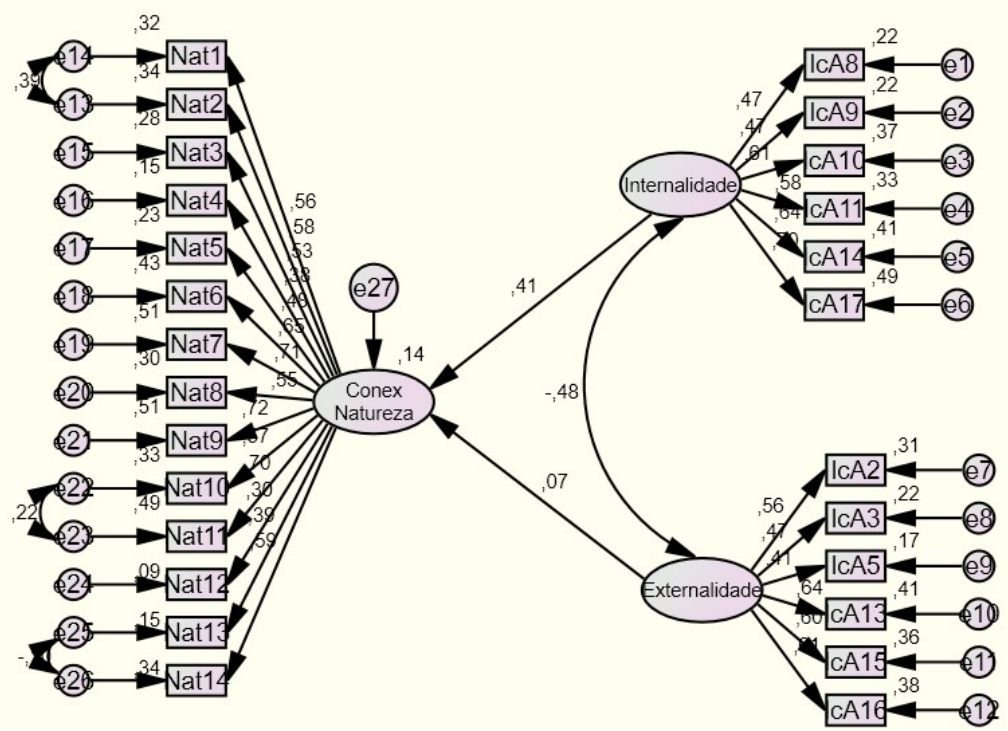

\section{Tabela 3}

Índices de ajuste do modelo

\begin{tabular}{cccc}
\hline Indicadores & $\begin{array}{c}\text { Valores com ajuste } \\
\text { de erros de } \\
\text { medida }\end{array}$ & $\begin{array}{c}\text { Valores sem ajuste } \\
\text { de erros de medida }\end{array}$ & Valor ideal \\
\hline $\mathrm{X}^{2}$ & 472,715 & 571,923 & - \\
$\mathrm{X}^{2} / \mathrm{gl}$ & 2,110 & 2,519 & Até 5 \\
$\mathrm{RMR}$ & 0,039 & 0,041 & Menor que 0,05 \\
GFI & 0,918 & 0,901 & Acima de 0,80 \\
AGFI & 0,899 & 0,880 & Acima de 0,80 \\
CFI & 0,911 & 0,877 & Próximo a 1 \\
RMSEA & $0,049(0,043-$ & $0,058(0,052-$ & Menor que 0,10 \\
& $0,056)$ & $0,064)$ & \\
\hline
\end{tabular}


A lógica defendida no estudo foi parcialmente confirmada, uma vez que, de fato, foi observada uma influência positiva significativa da internalidade sobre a conexão com a natureza. Entretanto, no que se refere à externalidade, tal influência não foi significativa. A correlação observada entre locus interno e externo foi negativa e significativa ( $p=0,000)$.

Os resultados obtidos são de difícil discussão, a partir dos resultados de outros estudos, tendo em vista não haver instrumento correspondente na literatura, o que se configura também como uma limitação deste estudo, mas é justamente isso sua maior contribuição para a área.

Entretanto, considerando estudos tanto relacionados com a área ambiental como com - locus de controle, é possível considerar que os resultados do presente estudo são condizentes com o que vem sendo preconizado pela literatura, principalmente diante da constatação de que indivíduos internos possuem a forte crença de que o alcance de seus objetivos dependerá em grande parte de suas próprias ações. De fato, Bandura (1986) defende que o locus de controle é uma variável central na explicação de aspectos cognitivos, afetivos, comportamentais e fisiológicos relacionados ao indivíduo.

Desde as sistematizações iniciais do estudo do locus de controle, diversos estudos têm sido realizados, considerando diferentes áreas do conhecimento e contextos bastante variados, para explicar como indivíduos internos possuem maior probabilidade de sucesso nas ações que executam. Indivíduos externos, por sua vez, sequer se propõem a buscar atuar sobre o ambiente por acreditarem que outras forças são determinantes para que as coisas ocorram dessa ou daquela forma. Seguindo tal lógica, qualquer esforço de mudança da realidade será em vão.

É nesse sentido que o locus de controle tem sido investigado para explicar comportamentos associados à saúde, estilos de liderança, satisfação no trabalho, adaptabilidade de carreira, atitude e comportamento pró-ambiental (Asghar \& Nasneen, 2016; Dumitriu, Timofti, Nechita, \& Dumitriu, 2014; Kim \& Lee, 2018; Lima, Muniz, Salome, \& Ferreira, 2018; Singh, Singh, \& Gupta, 2018), entre diversos outros temas. A lista seria certamente extensa e improdutiva em seu esforço em demonstrar a extensa variedade de temas que têm sido associados ao locus de controle. Recomenda-se reconhecer que 
características individuais podem influenciar comportamentos relacionados ao ambiente. (Martinsson \& Lundqvist, 2010).

\section{Conclusão}

O diagnóstico favorável individual na direção de um comportamento pró-ambiental usando locus de controle da conexão da natureza já é uma busca pelo resgate do ser humano urbanizado, que vive distante de suas origens e das fontes de recursos naturais, ao nascer e desenvolver-se em ambientes industrializados. Sem ser sensibilizadas, informadas e preparadas para o agravamento ambiental das condições que as cerca, as pessoas pouco poderão fazer, caso queiram. Tal vulnerabilidade precisa ser suportada por mais iniciativas que ajudem na transformação para um ser ético, responsável ambientalmente e gerente do futuro dos outros.

\section{Referências}

Asghar, M., \& Nazneen, L. (2016). The Role of Locus of Control in Pro Environmental Attitude and Behavior in Youth. Peshawar Journal of Psychology and Behavioral Sciences, 2(2), 263-274. https://doi.org/10.32879/pjpbs.2016.2.2.263-272

Bandeira, M., Quaglia, M. A. C., Bachetti, L. D. S., Ferreira, T. L., \& Souza, G. G. D. (2005). Comportamento assertivo e sua relação com ansiedade, locus de controle e autoestima em estudantes universitários. Estudos de Psicologia (Campinas), 22(2), 111-121. http://doi.org/10.1590/S0103-166X2005000200001

Bandura, A. (1986). Social foundations of thought and action. Englewood Cliffs: Prentice Hall.

Byrne, B. M. (2001). Structural equation modeling with AMOS. Mahwah, NJ: Lawrence Erlbaum

Capaldi, C. A., Dopko, R. L., \& Zelenski, J. M. (2014). The relationship between nature connectedness and happiness: a meta-analysis. Frontiers in Psychology, 5, 1-15. https://doi.org/10.3389/fpsyg.2014.00976 
Clarke, S., Probst, T. M., Guldenmund, F. W., \& Passmore, J. (2015). The Wiley Blackwell Handbook of the Psychology of Occupational Safety and Workplace Health. New York: John Wiley \& Sons.

Coleta, M. F. D. (1987). Escala multidimensional de locus de controle de Levenson. Arquivos

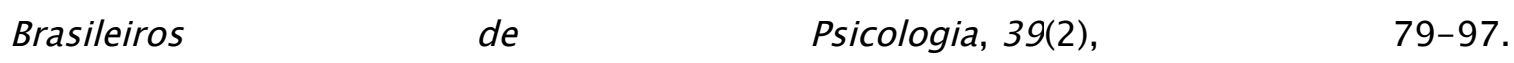
http://bibliotecadigital.fgv.br/ojs/index.php/abp/article/view/19592

Corral-Verdugo, V. (2012). The positive psychology of sustainability. Environment, Development and Sustainability, 14, 651-666. https://doi.org/10.1007/s10668-012$9346-8$

Damerell, P., Howe, C., \& Milner-Gulland, E. J. (2013). Child-orientated environmental education influences adult knowledge and household behaviour. Environmental Research Letters, 8(1), 015016. https://doi.org/10.1088/1748-9326/8/1/015016

Dumitriu, C., Timofti, L. C., Nechita, E., \& Dumitriu, G. (2014) The Influence of the Locus of Control and Decision-making Capacity upon the Leadership Style. Social and Behavioral Sciences, 141, 494-499. https://doi.org/10.1016/j.sbspro.2014.05.086

Flammer, C. (2013). Corporate social responsibility and shareholder reaction: The environmental awareness of investors. Academy of Management Journal, 56(3), 758781. https://doi.org/10.5465/amj.2011.0744

Global Footprint Network (2018). National footprint accounts. 2018 edition http://data. footprintnetwork.org.

Hair, J. F., Black, W. C., Babin, B. J., Anderson, R. E., \& Tatham, R. L. (2006) Multivariate Data Analysis. Vol. 6, Pearson Prentice Hall, Upper Saddle River.

Howell, A. J., Dopko, R. L., Passmore, H., Buro, K. (2011). Nature connectedness: Associations with well-being and mindfulness. Personality and Individual Differences, 51, 166-171. https://doi.org/10.1016/j.paid.2011.03.037Kaplan, D. (2000). Structural Equation Modeling: foundations and extensions Sage: Thousand Oaks.

Karch, P. (2002). Environmental Awareness in Germany and USA: A Comparative Study of Two Selected Student Bodies [Unpublished master's thesis]. University of Southern California. 
Kim, N. R., \& Lee, K. H. (2018). The effect of internal locus of control on career adaptability: the mediating role of career decision-making self-efficacy and occupational engagement. Journal of Employment Counseling, 55(1), 2-15. https://doi.org/10.1002/joec. 12069

Kortenkamp, K. V., \& Moore, C. F. (2001). Ecocentrism and Anthopocentrism: Moral reasoning about ecological commons dilemmas. Journal of Environmental Psychology, 21(3), 1-13. https://doi.org/10.1006/jevp.2001.0205

Le Blanc, D. (2015). Towards integration at last? The sustainable development goals as a network of targets. Sustainable Development, 23(3), 176-187. https://www.un.org/esa/desa/papers/2015/wp141_2015.pdf

Levenson, H. (1981). Differentiating among internality, powerful others, and chance. In H. M. Lefcourt (Ed.), Research with the Locus of Control Construct (pp. 15-63). New York: Academic Press.

Lima, J. A., Muniz, K. C., Salome, G. M., \& Ferreira, L. M. (2018). Association of sociodemographic and clinical factors with self-image, self-esteem and locus of health control in patients with an intestinal stoma. Journal of Coloproctology, 38(1), 56-64. https://doi.org/10.1016/j.jcol.2017.11.003

Martinsson, J., \& Lundqvist, L. J. (2010). Ecological citizenship: coming out 'clean' without turning 'green'?. Environmental Politics, 19(4), 518-537.

Mayer, F. S., \&Frantz, C. M. (2004). The connectedness to nature scale: a measure of individuals' feeling in community with nature. Journal of Environmental Psychology, 24, 503-515. https://doi.org/10.1016/j.jenvp.2004.10.001

Nisbet, E. K., Zelenski, J. M., \& Murphy, S. A. (2011). Happiness is in our nature: Exploring nature relatedness as a contributor to subjective well-being. Journal of Happiness Studies, 13, 303-322. https://doi.org/10.1007/s10902-010-9197-7

ONU (2018). World Urbanization Prospects: The 2018 Revision. Department of Economic and Social Affairs, Online Edition. Disponível em: https://bit.ly/37UDeCk. Acessado em 22 jun. 2018. 
Pessoa, V. S.; Gouveia, V. V.; Soares, A. K. S.; Vilar, R., \& Freires, L.A. (2016) . Escala de conexão com a natureza: evidências psicométricas no contexto brasileiro. Estudos de Psicologia (Campinas), 33(2), 271-282. doi: 10.1590/1982-02752016000200009

Quinn, C., \& Burbach, M. E. (2008). Personal characteristics preceding pro-environmental behaviors que improve surface water quality. Great Plains Research, 18, 103-114.

Robbins, S. P. (2005). Comportamento organizacional $\left(11^{\text {a }}\right.$ ed.). São Paulo: Prentice Hall Brasil.

Rotter, J. (201 1). Rotter Internal-External Locus of Control Scale. New York: Springer

Singh, A. P., Singh, A. K., \& Gupta, V. K. (2018). Role of life events stress and locus of control (external) in job satisfaction: An empirical evidence. Indian Journal of Positive Psychology, 9(1), 69-73. https://doi.org/10.15614/ijpp.v9i01.11745

Steg, L., Bolderdijk, J. W., Keizer, K., \& Perlaviciute, G. (2014). An integrated framework for encouraging pro-environmental behaviour: The role of values, situational factors and goals. Journal of Environmental Psychology, 38, 104115. https://doi.org/10.1016/j.jenvp.2014.01.002

Tamayo, A. (2012). Validade fatorial da escala Levenson de locus de controle. Psicologia: $\begin{array}{llll}\text { Teoria } & \text { Pesquisa, } & \text { 5(1), } & 111-122 .\end{array}$ https://periodicos.unb.br/index.php/revistaptp/article/view/17063

Tan-Mullins, M., \& Mohan, G. (2013). The potential of corporate environmental responsibility of Chinese state-owned enterprises in Africa. Environment, Development and Sustainability, 15(2), 265-284. https://doi.org/10.1007/s10668-012-9409-x

Ullman, J.B. (2007). Structural equation modeling. In Tabachnick, B.G. and Fidell, L.S. (Eds), Using Multivariate Statistics, 5a. ed., Pearson Education, Boston, MA, pp. 676-780.

VandenBos, G. R. (2015). APA dictionary of psychology. Washington: American Psychological Association.

Zedeck, S. (Ed.). (2011). APA handbook of industrial and organizational psychology, Vol 3: Maintaining, expanding, and contracting the organization. New York : American Psychological Association. https://doi.org/10.1037/12171-000 
Recebido em: 22/8/2018 Aprovado em: 22/3/2019 